\title{
Real-time monitoring of the evolving morphology and molecular structure at an organic-inorganic semiconductor interface: $\mathrm{SnPc}$ on $\mathrm{GaAs}(001)$
}

\author{
D. A. Evans, ${ }^{\text {a) }}$ A. R. Vearey-Roberts, O. R. Roberts, A. C. Brieva, ${ }^{\text {b) }}$ A. Bushell, ${ }^{c}$ \\ G. T. Williams, and D. P. Langstaff \\ Institute of Mathematics and Physics, Aberystwyth University, Aberystwyth SY23 3BZ, United Kingdom \\ G. Cabailh ${ }^{\text {d) }}$ and I. T. McGovern ${ }^{\text {d) }}$ \\ School of Physics, Trinity College, Dublin 2, Ireland
}

(Received 9 March 2010; accepted 28 June 2010; published 27 July 2010)

\begin{abstract}
An organic-III-V hybrid semiconductor interface has been studied using real-time photoelectron spectroscopy and x-ray absorption spectroscopy to reveal the evolving morphology and molecular structure within the organic layer during thin film growth. This new approach to in situ characterization has been enabled by electron detection using a direct electron-counting array detector coupled to a hemispherical electron analyzer. The nonplanar tin phthalocyanine (SnPc) molecules initially form a uniform layer within which they have a distinct molecular orientation relative to the S-passivated gallium arsenide substrate surface $[\mathrm{GaAs}: \mathrm{S}(001)]$. The critical thickness of $0.9 \mathrm{~nm}$ that marks the transition between layered and clustered growth, determined from the photoemission measurements, corresponds to a single molecular layer with the molecules oriented at an angle of $(39 \pm 2)^{\circ}$ to the substrate plane. This value is confirmed by angle-resolved near-edge $\mathrm{x}$-ray absorption fine structure measurements in the same experimental environment. However, the angle is less for the thicker films as the molecule-molecule interaction dominates over the molecule-substrate interaction and the structure is close to that of the bulk triclinic SnPc crystal. (C) 2010 American Vacuum Society. [DOI: 10.1116/1.3464769]
\end{abstract}

\section{INTRODUCTION}

Organic semiconductors offer low cost and low toxicity options for optoelectronic and electronic applications where these factors offset their lower inherent performance compared to crystalline inorganic optoelectronic materials. Of the organic semiconductors currently in use, the highest structural and electronic quality is found in ordered crystalline films of small conjugated molecules. ${ }^{1}$ They can be grown with high purity and structural quality, most commonly by vacuum deposition, but they exhibit a range of bulk and thin film crystal structures that are very process-sensitive.

Metal phthalocyanines, materials that are finding application in areas such as photovoltaics ${ }^{2}$ and sensors, ${ }^{3}$ commonly exhibit at least two structures ( $\alpha$ and $\beta$ ) when grown as bulk crystals, differing in the molecular orientation. ${ }^{4}$ There are also differences in the bulk structure for planar and nonplanar phthalocyanines. ${ }^{5}$ The molecular stacking is known to influence the electron transport properties in bulk materials, ${ }^{4}$ and the molecular structure in thin films has been shown to be dependent on temperature, ${ }^{4,6,7}$ substrate, ${ }^{8-10}$ film thickness, ${ }^{9}$ and growth rate. ${ }^{10}$ For nonplanar phthalocyanines such as tin phthalocyanine ( $\mathrm{SnPc})$, the molecules can order with the metal ion above or below the plane in the film depending on the growth conditions. ${ }^{7}$

\footnotetext{
a) Author to whom correspondence should be addressed; electronic mail: a.evans@aber.ac.uk

${ }^{b}$ Present address: University of Newcastle upon Tyne, Newcastle, UK

${ }^{c}$ Present address: Thermo VG Scientific, East Grinstead, Sussex, UK.

${ }^{d)}$ Present address: London Centre for Nanotechnology, University College London, UK.
}

In characterizing the growth of organic thin films, it is important to correlate structural, electronic, and chemical properties at the molecular level with the electrical and optical performance of the devices. A few techniques can provide all the requisite information in situ, but one technique that is able to provide much parallel information (on bonding, morphology, and energetics) is photoelectron spectroscopy, ${ }^{11}$ but this is not usually applied as an in situ tool due to the time necessary for data collection and the need for a vacuum environment. To address this, there have been many attempts to improve data collection efficiency using multichannel detection combined with bright x-ray and UV sources. These have been successfully applied to study surface processes for inorganic substrates ${ }^{12,13}$ but have not been applied to date as an in situ tool for monitoring the growth of organic semiconductor thin films and interfaces.

Electron detection systems for rapidly measuring both high and low intensity spectral features that evolve in time require a wide dynamic range and high sensitivity. We have developed a multichannel array detector that fulfills these requirements and that has been applied with both laboratory and synchrotron $\mathrm{x}$-ray sources to study surface processes in real time, ${ }^{14,15}$ and this has now been incorporated into an organic molecular beam deposition system as a real-time in situ probe of the growth of multilayer organic-inorganic device structures.

The interface selected for this study is $\mathrm{SnPc}$ on the S-passivated $\mathrm{GaAs}(001)$ surface. SnPc is currently applied, for example, in photovoltaic structures that are among the most efficient organic devices. ${ }^{1}$ III-V materials have attracted renewed interest due to their application in concentrator pho- 
tovoltaic cells, ${ }^{16}$ in addition to their more traditional applications in electronics and optoelectronics. Hybrid structures are less well developed, but it has, for example, been shown that nanometer-scale SnPc thin films can significantly modify the Fermi level in GaAs diodes in a way which has eluded efforts using surface preparation and metal contacts. ${ }^{17}$

The SnPc-GaAs system is thus a useful model system for this new experimental approach in that it provides an applications-relevant system where high-purity, controlled thin films can be grown on a well-characterized, passivated substrate surface in an environment that enables fast photoelectron spectroscopy to be applied in situ and in real time.

\section{EXPERIMENT}

Organic molecules were thermally evaporated from a water-cooled Knudsen cell heated to around $350{ }^{\circ} \mathrm{C}$ located within the ultrahigh vacuum spectrometer. The growth rate was determined by a quartz crystal microbalance placed near the substrate, such that growth rates of $0.05-1.0 \mathrm{~nm} \mathrm{~min} \mathrm{~m}^{-1}$ could be obtained while maintaining the system pressure at $<10^{-9}$ mbar. Passivated $n$-type and $p$-type GaAs(001) substrates were prepared by etching epi-ready wafers (Freiberger) in dilute $\mathrm{S}_{2} \mathrm{Cl}_{2}$ solution and then annealing in vacuum to $450{ }^{\circ} \mathrm{C}$. This produces a passivated, ordered surface that is oxygen-free and contains a surface layer of $\mathrm{Ga}$ and $\mathrm{S}$ atoms with a $2 \times 1$ structure with respect to the bulkterminated $\operatorname{GaAs}(001)$ lattice. ${ }^{17,18}$

Photoexcitation was provided by soft $\mathrm{x}$ rays from bending magnet beamlines at the UK Synchrotron Radiation Source, and the photoelectrons were collected and energy analyzed using a hemispherical analyzer equipped with a 768-channel array detector positioned behind a multichannel plate at the focal plane of the analyzer. This $19 \mathrm{~mm}$ device was used to image energy windows of 5-10 eV that enabled core level photoelectron spectra (such as the Ga $3 d$ and As $3 d$ levels of GaAs) to be recorded without scanning the hemisphere potential. Using synchrotron radiation excitation, it was possible to record such spectra as rapidly as $250 \mathrm{~ms}$, with a total count rate across the array of around $4 \mathrm{MHz}$.

In initial thin film growth experiments, several regions, including low intensity band edges, were recorded sequentially using longer time intervals of $1-10 \mathrm{~s}$ for each spectral region. This was found to be adequate for the low growth rates of $\sim 0.1 \mathrm{~nm} \mathrm{~min}^{-1}$ used in these experiments. For the most accurate probe of the growth mode, single core level spectra were recorded in separate multiple growth runs. In this mode, all analyzer and detector parameters, K-cell parameters, beam settings, and the sample position were kept constant during growth. For wide energy range characterization spectra and higher energy resolution spectra, the electron detector was operated in a conventional way by scanning the electron energy.

These photoemission measurements provide insights into interface bonding and interface energetics, but the focus in this paper is on the thin film morphology that is revealed by the intensity variation of the substrate and overlayer core levels. Probing molecular order within the films was possible
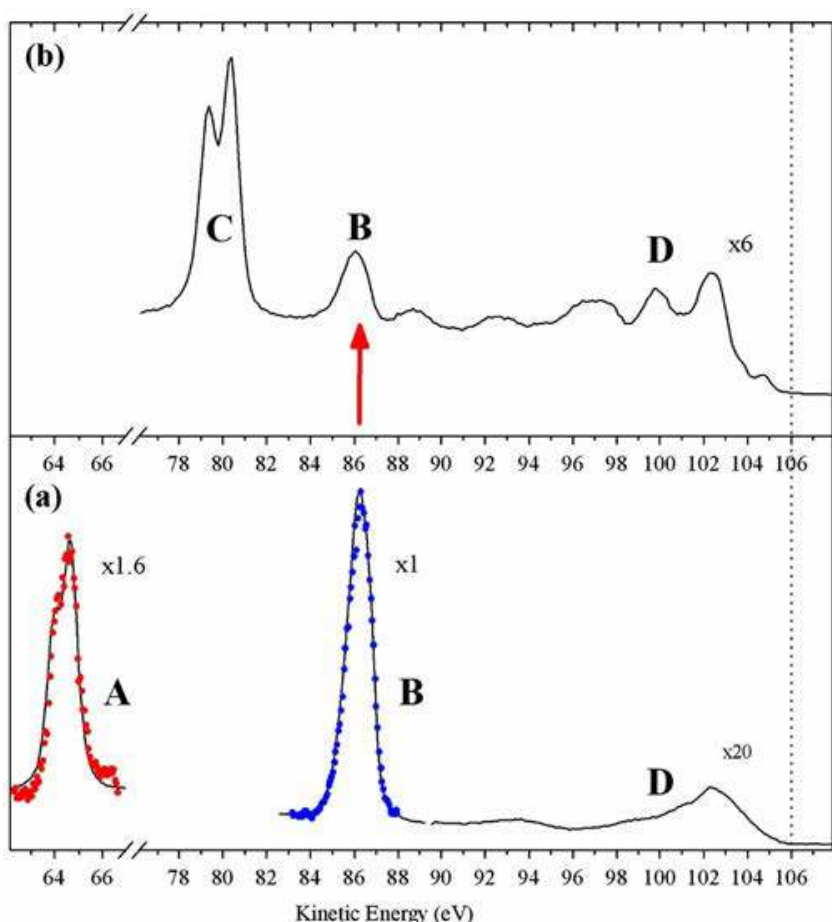

FIG. 1. (Color online) Photoelectron spectra (a) for the GaAs:S(001) surface and (b) following adsorption of a thin film of SnPc. Features shown represent the substrate As $3 d$ and Ga $3 d$ core levels (A and B, respectively), (C) the $\mathrm{Sn} 4 d$ core level of the central metal ion in the SnPc molecule, and the valence band/molecular orbital region (D). The dotted line represents the Fermi level. Rapid data collection is illustrated by the snapshot spectra (filled symbols) recorded in $250 \mathrm{~ms}$ for the (A) As $3 d$ and (B) Ga $3 d$ core levels.

in the same growth/characterization environment by measuring the near-edge $\mathrm{x}$-ray absorption fine structure (NEXAFS) at the $\mathrm{C}$ and $\mathrm{N} K$-edge of the organic molecules. Spectra were recorded in total electron yield mode at selected film thicknesses informed by the real-time photoelectron spectroscopy measurements. The planar nature of the molecule enables the angular dependence of the core- $\pi^{*}$ resonance features in the NEXAFS spectra to be used to determine the molecular orientation within the film.

Atomic force microscopy (AFM) images of the grown organic films were recorded ex situ in noncontact dynamic force mode using a Park Systems XE-100 microscope. Noncontact was found to be essential as the tip was found to cause disruption of the organic film in contact mode.

\section{RESULTS AND DISCUSSION}

Spectra recorded in conventional scanned mode are shown (solid lines) in Fig. 1 (a) for the clean, S-passivated $\mathrm{GaAs}(001)$ surface and (b) for a thin $(5 \mathrm{~nm})$ film of SnPc. Also shown (filled circles) are $250 \mathrm{~ms}$ snapshot spectra for the substrate $\mathrm{Ga} 3 d$ and As $3 d$ core levels. Although somewhat compromising energy resolution, such snapshot spectra accurately reflect the changes in intensity that are of principal interest here. The energy resolution is important for systems where there are strong chemical changes, but in this case, it is known that the passivated GaAs surface is chemi- 
(a)

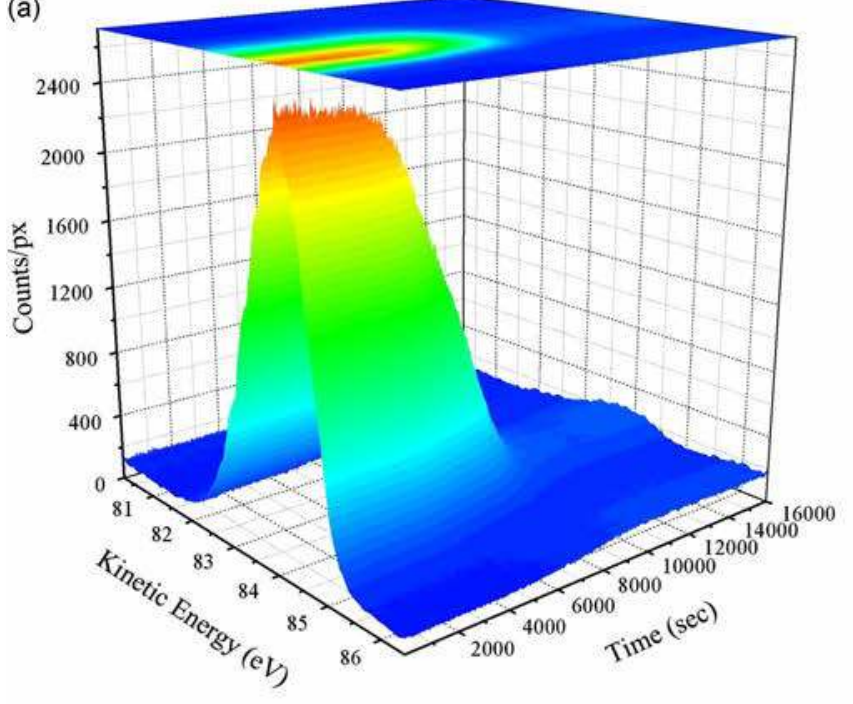

(b)

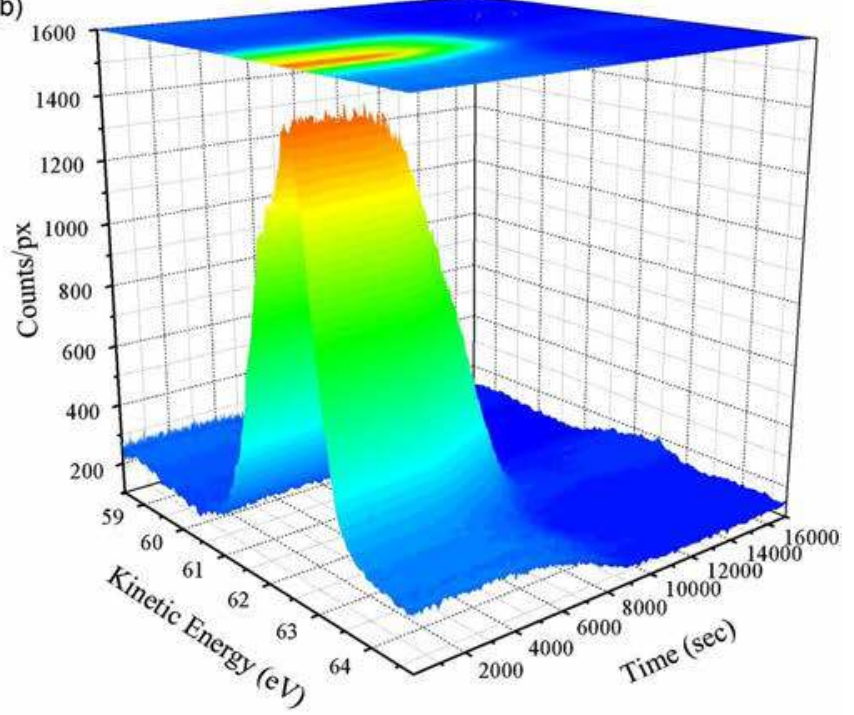

(c)

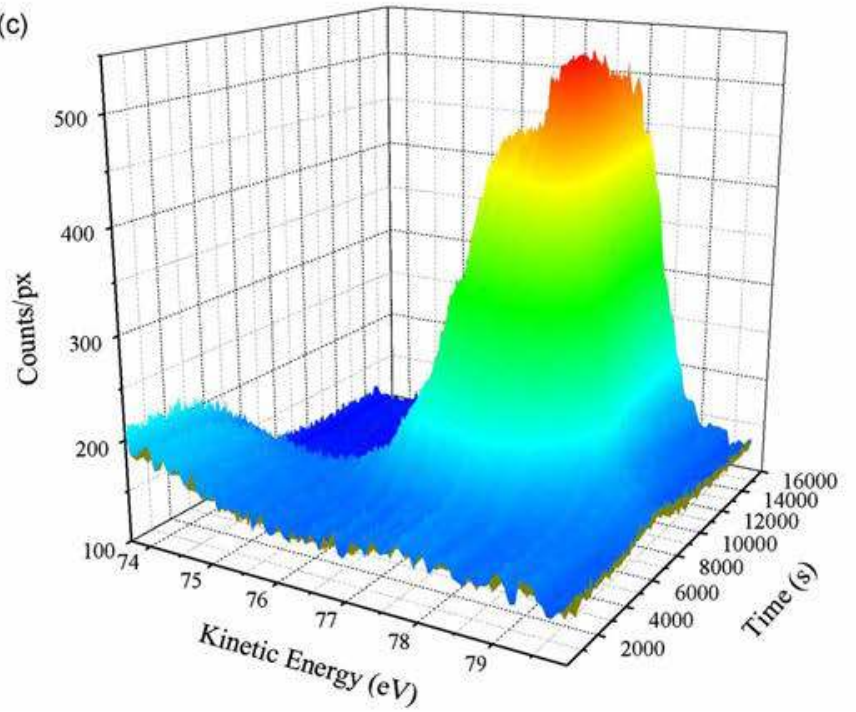

FIG. 2. (Color online) Core level spectra recorded in real time during the growth of SnPc on GaAs:S(001) at $300 \mathrm{~K}$. The substrate (a) Ga $3 d$ and (b) As $3 d$ spectra and (c) the overlayer Sn $4 d$ level were recorded sequentially with a constant photon energy of $106 \mathrm{eV}$.

cally inactive when exposed to metal phthalocyanines, and this is reflected in the absence of significant line-shape changes in the substrate core levels during thin film growth. ${ }^{18}$ In addition to changes in intensity, there are rigid shifts in the core levels related to organic-induced changes in the interface energetics that have been used to explain the variation in the barrier height of organic-modified $n$-GaAs Schottky diodes. ${ }^{17}$ The photon energy was selected to ensure surface sensitivity for all core levels while retaining sufficient photoelectron intensity at the band edge. This sensitivity enabled the accurate in situ monitoring of the initial (monolayer) stages of growth.

Sequential core level spectra collected in real time during exposure of the GaAs:S(001) surface to the $\mathrm{SnPc}$ flux are shown in Fig. 2. Each spectrum was recorded in snapshot mode in $1 \mathrm{~s}$ intervals as images on the array detector. The data have been corrected only for array nonuniformity ${ }^{19}$ and are shown integrated in eight-channel intervals in the energy domain. The data are representative of many experiments where the rapid data collection allows many repeats; the growth of a $5 \mathrm{~nm} \mathrm{SnPc}$ layer could, for example, be fully monitored in $1 \mathrm{~h}$.

For the first $2000 \mathrm{~s}$, spectra were recorded for the clean GaAs:S(001) surface to ensure incident beam stability and this is confirmed by the unchanged As $3 d$ and Ga $3 d$ core level intensities (and peak positions) in Fig. 2. At 2000 s, the $\mathrm{SnPc}$ cell was switched on and, as the evaporation temperature is reached, both substrate core levels show a decrease in intensity, with a corresponding increase in the Sn $4 d$ peak intensity.

Fitting of the core level data provides the time (coverage) evolution of the core level peak intensities as shown in Fig. 3 . The attenuation rate of both substrate core levels increases in region $\mathrm{A}$ as the $\mathrm{SnPc}$ flux increases from zero at $2000 \mathrm{~s}$ up to its maximum, stable rate of $0.1 \mathrm{~nm} \mathrm{~min}^{-1}$ at $t=6000 \mathrm{~s}$. Between 6000 and $8000 \mathrm{~s}$, the attenuation rates are at their 


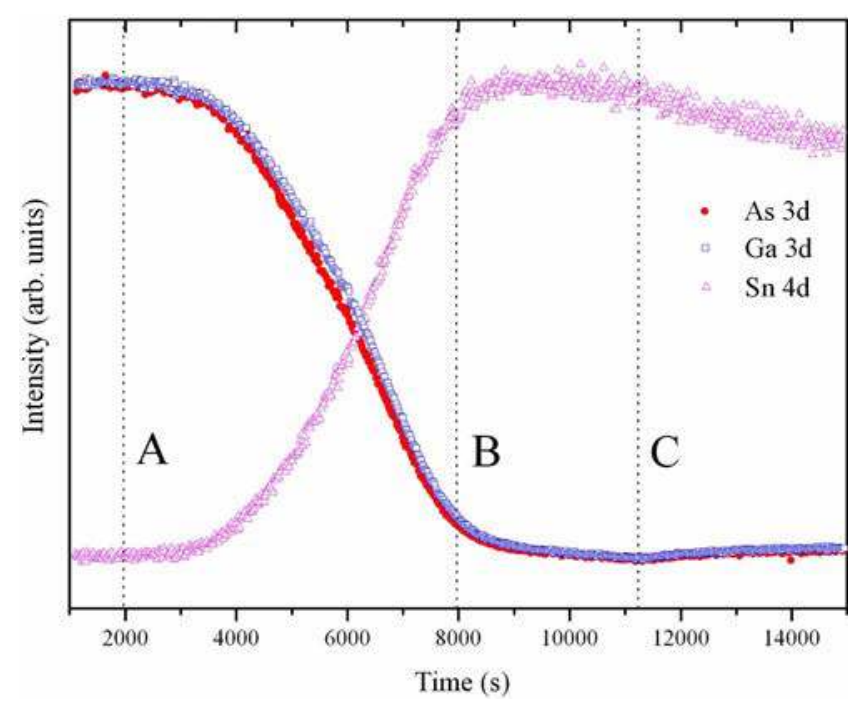

FIG. 3. (Color online) Variation of the fitted core level peak intensities during SnPc growth. Three regions are revealed: (A) uniform growth, (B) clustered growth, and $(\mathrm{C})$ postgrowth reorganization.

highest before decreasing significantly beyond $8000 \mathrm{~s}$ up to the cell switch-off point at $11200 \mathrm{~s}$ (region B). There is a slight difference in the attenuation of the Ga $3 d$ and As $3 d$ core levels due to the different photoelectron mean free path. The transition between regions $\mathrm{A}$ and $\mathrm{B}$ is indicative of a Stranski-Krastanov (layer+island) growth mode. Beyond $11200 \mathrm{~s}$ (region C), there is a small recovery of the substrate core level intensities and a continued decrease in the Sn $4 d$ core level intensity that is associated with a timedependent molecular reorganization that is currently under further study.

In a series of experiments probing individual core level spectra, the SnPc K-cell was controlled using a manual shutter to ensure a linear growth rate $\left(0.1 \mathrm{~nm} \mathrm{~min}^{-1}\right)$ throughout the exposure. This rate, calibrated using a quartz crystal monitor, was used to convert the time of exposure to thin film thickness as shown in Fig. 4 for the As $3 d$ core level attenuation during exposure of the GaAs:S(001) surface to SnPc at room temperature. The intensity, plotted on a logarithmic scale, shows an exponential decrease in region A and this was fitted (dotted line in Fig. 4) to a model function $I$ $=I_{0} \exp \lfloor-x / \lambda\rfloor$ to give a value for the electron mean free path, $\lambda=0.34 \mathrm{~nm}$. The critical thickness $x_{c}$ that marks the transition between layered (region A) and island (region B) growth determined from these data is found to be $x_{c}=0.9 \mathrm{~nm}$. This value is smaller than that previously reported for this interface based on less accurate, conventionally recorded values. ${ }^{20}$ This behavior is consistent with a StranskiKrastanov (layer+island) growth mode, and the clustered nature of the organic film has been confirmed using AFM. Due to the low electron mean free path, the substrate photoelectron emission peaks are attenuated significantly by $\mathrm{SnPc}$ films of nanometer thickness and the spectra are essentially those of bulk SnPc at $5 \mathrm{~nm}$. There is thus an inherent efficiency in terms of material used-a near-complete picture of

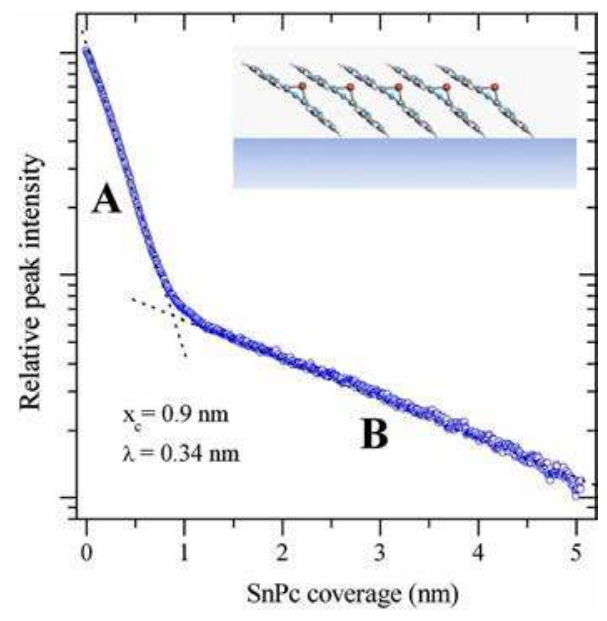

FIG. 4. (Color online) Semilogarithmic plot of the As $3 d$ core level peak intensity against $\mathrm{SnPc}$ coverage, illustrating the transition from (A) uniform to (B) clustered growth.

the interface in terms of energy bands and bonding is extractable with a fraction of the material required for a single electronic or optoelectronic device.

If it is assumed that the uniform layer (region A in Fig. 4) is made up of a single layer of stacked molecules, then the molecular orientation can be estimated from this value of critical thickness. The in-plane molecular dimensions based on the calculated $\mathrm{SnPc}$ structure are around $1.3 \mathrm{~nm}$ along the benzene ring axis and around $1.5 \mathrm{~nm}$ for the diagonal along the benzene ring-metal ion-benzene ring axis. However, in monolayer absorption measurements, the benzene ring axis dimension allowing for the electron density at the outer hydrogen atoms is given as $d=1.4 \mathrm{~nm} .{ }^{21}$ In the absence of any direct evidence for the in-plane molecular orientation, a value of $d=1.4 \mathrm{~nm}$ is taken to calculate the orientation of the $\mathrm{SnPc}$ molecules with respect to the GaAs surfaces. The angle obtained is $\theta=\sin ^{-1}\left(x_{c} / d\right)=(39 \pm 2)^{\circ}$. The predicted molecular orientation within this first layer is illustrated in the inset of Fig. 4. The calculated structure of the $\mathrm{SnPc}$ molecule shows that the central $\mathrm{Sn}$ ion displaced out of the ligand plane, but this does not severely distort the planarity of the latter.

In order to investigate further the molecular orientation within the SnPc thin film, NEXAFS measurements were performed on selected thin films. N $K$-edge spectra showing near-edge $\mathrm{N} 1 s \rightarrow \pi^{*}$ and $\sigma^{*}$ resonances for varying film thickness are shown in Fig. 5. Spectra a, b, and c correspond to thicknesses below, at, and above the critical thickness, respectively. The NEXAFS spectra have been corrected to the incidence flux intensity and normalized on either side of the absorption edge. They are shown with equal height for clarity and each spectrum was recorded with an angle of $70^{\circ}$ between the substrate and the incident x-ray beam. The spectral features of the $\mathrm{N} K$-edge spectra are similar to those for various metal phthalocyanines, indicating that the lowest unoccupied molecular orbital levels are derived mainly from the organic ligand rather than the central metal atom. ${ }^{22}$ By comparison, the NEXAFS for a fluorinated phthalocyanine 


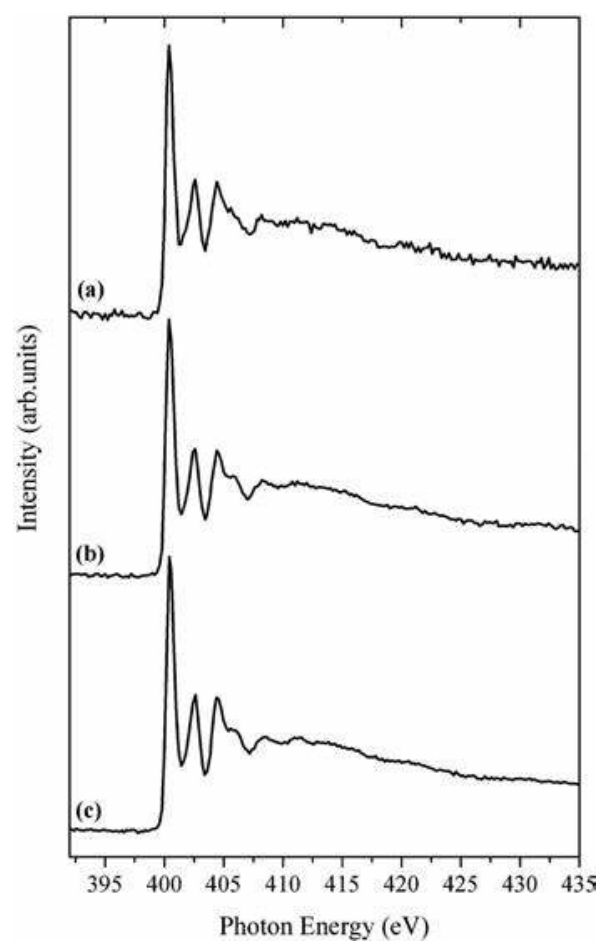

FIG. 5. N $K$-edge NEXAFS spectra recorded at a substrate angle of $70^{\circ}$ for SnPc films of thickness (a) $0.4 \mathrm{~nm}$, (b) $0.8 \mathrm{~nm}$, and (c) $4 \mathrm{~nm}$.

where the outer $\mathrm{H}$ atoms are replaced by $\mathrm{F}$ atoms is markedly different. $^{23}$ There is consensus that the spectral features nearest the absorption edge are largely due to core- $\pi^{*}$ resonances while the higher energy resonances are more $\sigma^{*}$-like in character. $^{24,25}$ Due to the planar nature of the molecule and the linear polarization of the synchrotron x-ray beam, the relative intensities of these resonance features can be used to probe the molecular orientation as well as the energy distribution of the unoccupied molecular orbitals.

In the spectra presented in Fig. 5, the number of peaks and their position and shape do not alter from a thin (monolayer) layer to a thicker (multilayer) layer, confirming that there is little reaction between the phthalocyanine overlayer and the S:GaAs substrate. The carbon $K$-edge spectra (not shown) are also unchanged in line shape for these film thicknesses. The relative intensities of the resonance peaks above and below the critical thickness are also similar, indicating that there is not a strong change in molecular orientation as the film makes the transition from an ordered initial layer to clustered growth in thicker films.

The molecular orientation can be quantified by measuring the angular dependence of the $\mathrm{N} K$-edge NEXAFS as shown in Fig. 6 for a $4 \mathrm{~nm} \mathrm{SnPc} \mathrm{film.} \mathrm{There} \mathrm{is} \mathrm{a} \mathrm{strong} \mathrm{angular}$ variation in the relative intensities of the $\mathrm{N} 1 s \rightarrow \pi^{*}$ and $\sigma^{*}$ resonances that reflects a preferred orientation of the molecule with respect to the substrate. When the field vector of the incident beam light is parallel to the substrate surface (sample angle of $0^{\circ}$ in Fig. 6), the intensity of the $\pi^{*}$ resonance peaks is at its weakest and the $\sigma^{*}$ contributions at energies above $408 \mathrm{eV}$ dominate. As the substrate angle is increased, the field vector is brought into alignment with the

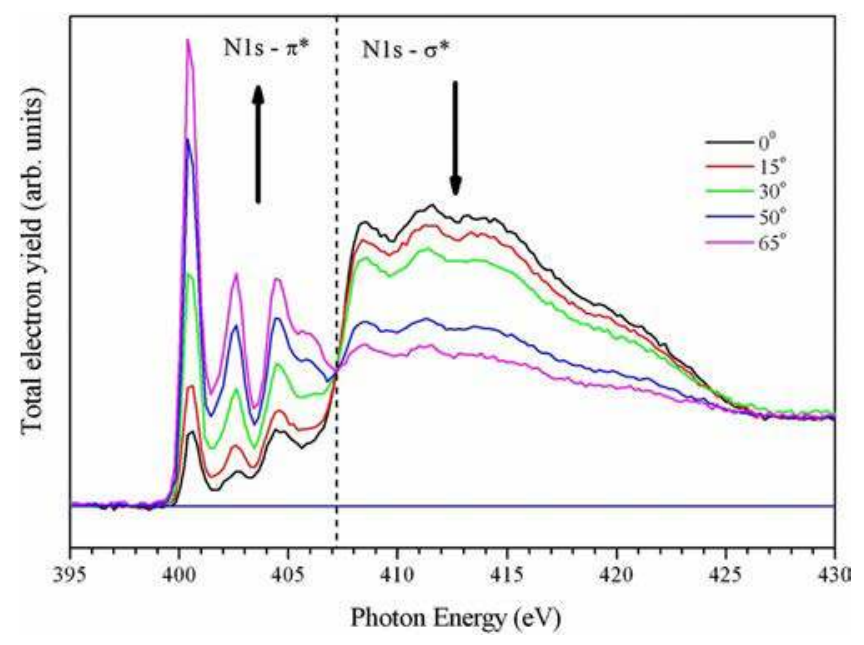

FIG. 6. (Color online) N $K$-edge NEXAFS spectra for a $0.8 \mathrm{~nm} \mathrm{SnPc} \mathrm{film} \mathrm{on}$ the GaAs:S(001) surface. Spectra have been normalized to the step edges. The strong angular dependence reveals a preferred molecular orientation relative to the substrate.

$\pi^{*}$ orbitals and the $1 s-\pi^{*}$ resonance features dominate. These data confirm previous studies that concluded that the molecules are preferentially aligned close to parallel to the surface. $^{20}$ The quality of these data, however, enables a more accurate value for the substrate angle to be determined.

The angular dependence of the intensity of the $1 s-\pi^{*}$ resonance of the phthalocyanine molecule is often modeled using the relationship $I \propto \cos ^{2}(\theta) .^{26}$ This, however, assumes that all the molecules are oriented at the same tilt angle and that the molecule is not rotated in the surface plane. Ellipsometry measurements on these films indicate that there is no molecular anisotropy in the plane of the GaAs substrate, and so it must be assumed that the molecule orientation is disordered azimuthally, but with a constant tilt angle. In this case, it has been shown ${ }^{26}$ that the angle dependence of the intensity can be modeled by Eq. (1) as follows:

$$
I \propto P\left(2 \cos ^{2}(\alpha) \cos ^{2}(\theta)+\sin ^{2}(\alpha) \sin ^{2}(\theta)\right)+(1-P) \sin ^{2}(\alpha),
$$

where $\alpha$ is the tilt angle of the molecular orbital vector with respect to the normal of the substrate surface, $\theta$ is the angle between the polarization vector of the incident beam and the surface, and $P$ is the degree of linear polarization of the x-ray beam.

Using Eq. (1), the angular dependence of the main $\mathrm{N} 1 s \rightarrow \pi^{*}$ transition intensities for three coverages has been computed by fitting to the experimental data as shown in Fig. 7. For the fitting procedure the polarization of the incident light was taken as $P=0.9$. The results of the fits yield angles of $(37 \pm 4)^{\circ}$ for the $0.4 \mathrm{~nm}$ film, $(33 \pm 3)^{\circ}$ for the $0.8 \mathrm{~nm}$ film, and $(28 \pm 3)^{\circ}$ for the $4 \mathrm{~nm}$ film. The uncertainties stated are calculated from the uncertainties of the fit and in measuring peak intensity from the NEXAFS data. The molecules are therefore lying close to parallel to the surface for both the monolayer and thicker films. This is common for flat substrates (AFM measurements confirm a substrate roughness of 


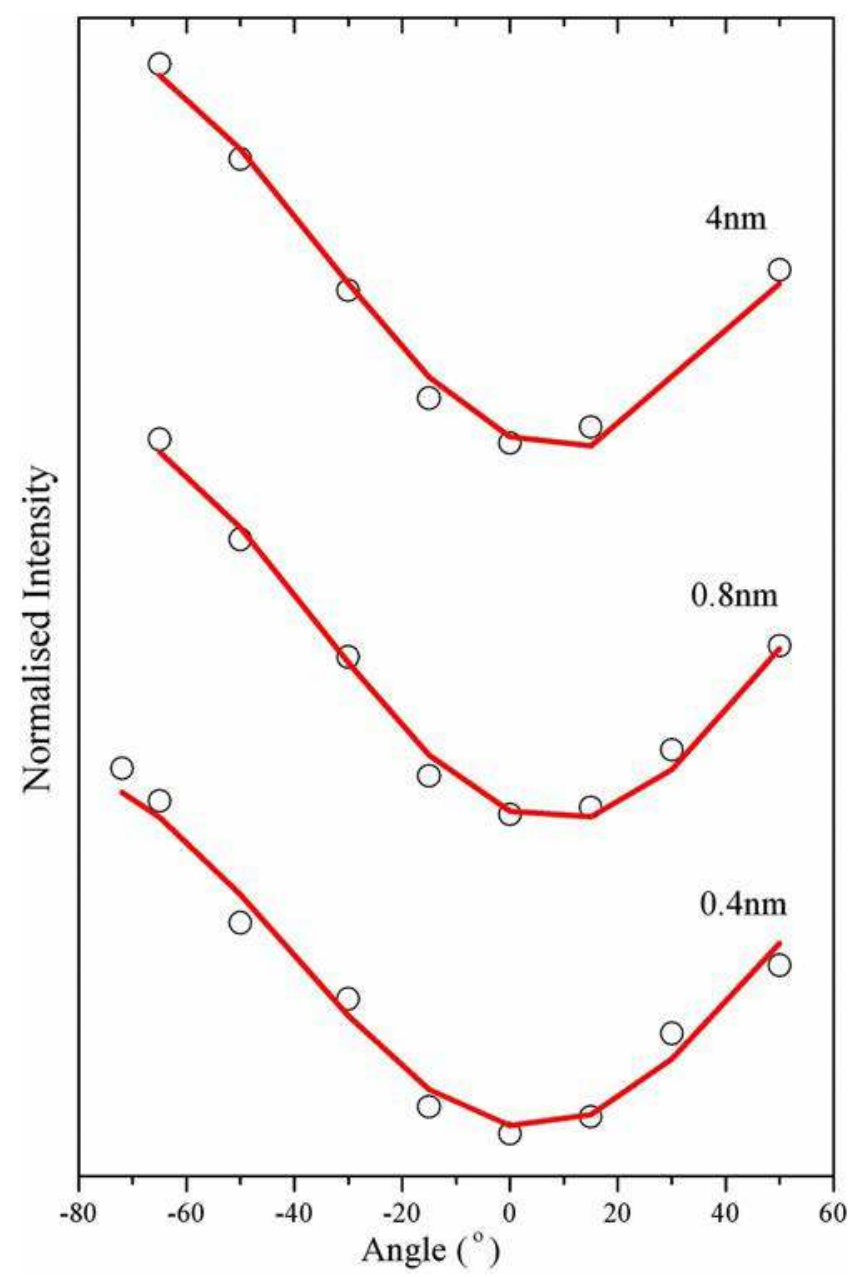

FIG. 7. (Color online) Fitted NEXAFS intensities for the main N $1 s-\pi^{*}$ resonance for three selected $\mathrm{SnPc}$ coverages.

around $0.7 \mathrm{~nm}$ ) but the orientation changes slightly for molecular films above the critical thickness, indicating a slight change in the growth structure as molecule-molecule interaction dominates over molecule-substrate interaction. The molecular orientation of the SnPc molecule in films of similar thicknesses on the $\operatorname{GaAs}(001) 1 \times 6$ substrate is similar and this is close to the orientation within the triclinic bulk phase of SnPc.

The angle determined for the $0.4 \mathrm{~nm}$ thin film is similar to that inferred from the photoemission measurements and this broadly supports the conclusion that the molecules arrange in a single layer up to the critical thickness. The agreement with the orientation within the $0.8 \mathrm{~nm}$ film is not as exact, lying just outside the error limits. This may be due to the large sampling areas, where variations in film growth are likely due to substrate surface inhomogeneity and local flux and temperature variation. The time taken between growth and measurement for the two techniques may also have an effect due to the molecular rearrangement that occurs over the time scales of such experiments.

Nevertheless, the data clearly show that the molecules are locked into a well-defined orientation within the first molecular layer even though there is no evidence for strong chemical bonding between the molecules and this surface. ${ }^{20}$ The van der Waals bonding between substrate and molecule dominates initially over molecule-molecule bonding, forcing the molecules into a single layer structure. Beyond this coverage, molecule-molecule attraction begins to dominate and, in region B in Fig. 3, there is competition between thin film growth and three-dimensional clustering. The time scales for growth and clustering are similar, and this results in a continued molecular clustering after exposure to the SnPc flux has ceased (region $\mathrm{C}$ in Fig. 3). AFM measurements confirm the formation of SnPc clusters on these surfaces, with clustering being enhanced further following removal from the growth chamber.

\section{CONCLUSIONS}

Photoelectron spectroscopy has been applied in real time to monitor the growth of $\mathrm{SnPc}$ on the passivated GaAs:S(001) surface. Intensity variations of substrate and overlayer core levels reveal a transition between layered and clustered growth at a critical thickness of $0.9 \mathrm{~nm}$ at room temperature. This corresponds to a single molecular layer oriented at an angle of $(39 \pm 2)^{\circ}$ to the substrate. This angle is in agreement with that determined from NEXAFS measurements on thin films of thickness less than the critical thickness $\left[\theta=(37 \pm 4)^{\circ}\right]$. Above this value, the molecules reorganize over time scales comparable to the growth rate and the angle is reduced to around $(28 \pm 3)^{\circ}$.

\section{ACKNOWLEDGMENTS}

This work was funded by the UK research councils (STFC, EPSRC-GB) and the EU, and was carried out within the Centre for Advanced Functional Materials and Devices, a HEFCW-funded Research and Enterprise partnership. Steve Sque and Jon Goss are thanked for the calculated structure of the SnPc molecule.

${ }^{1}$ S. Steudel, K. Myny, V. Arkhipov, C. Deibel, S. De Vusser, J. Genoe, and P. Heremans, Nature Mater. 4, 597 (2005).

${ }^{2}$ N. Li, B. E. Lassiter, R. R. Lunt, G. Wei, and S. R. Forrest, Appl. Phys. Lett. 94, 023307 (2009).

${ }^{3}$ B. Crone, A. Dodabalapur, A. Gelperin, L. Torsi, H. E. Katz, A. J. Lovinger, and Z. Bao, Appl. Phys. Lett. 78, 2229 (2001).

${ }^{4}$ R. D. Gould, Coord. Chem. Rev. 156, 237 (1996).

${ }^{5}$ M. K. Friedel, B. F. Hoskins, R. L. Martin, and S. A. Mason, J. Chem. Soc. D 400 (1970).

${ }^{6}$ N. Papageorgiou, E. Salomon, T. Angot, J. M. Layet, L. Giovanelli, and G. Le Lay, Prog. Surf. Sci. 77, 139 (2004).

${ }^{7}$ C. Stadler, S. Hansen, F. Pollinger, C. Kumpf, E. Umbach, T. L. Lee, and J. Zegenhagen, Phys. Rev. B 74, 035404 (2006).

${ }^{8}$ H. Peisert, T. Schwieger, J. M. Auerhammer, M. Knupfer, M. S. Golden, J. Fink, P. R. Bressler, and M. Mast, J. Appl. Phys. 90, 466 (2001).

${ }^{9}$ H. Peisert, I. Biswas, M. Knupfer, and T. Chassé, Phys. Status Solidi B 246, 1529 (2009).

${ }^{10}$ D. A. Evans et al., J. Phys.: Condens. Matter 15, S2729 (2003).

${ }^{11}$ D. A. Evans et al., Nucl. Instrum. Methods Phys. Res. B 199, 475 (2003).

${ }^{12}$ G. Paolucci, J. Phys.: Condens. Matter 13, 11293 (2001).

${ }^{13}$ A. Nambu et al., J. Electron Spectrosc. Relat. Phenom. 137-140, 691 (2004).

${ }^{14}$ D. P. Langstaff, A. Bushell, T. Chase, and D. A. Evans, Nucl. Instrum. Methods Phys. Res. B 238, 219 (2005).

${ }^{15}$ D. P. Langstaff, D. A. Evans, O. R. Roberts, and X. Zhu, Nucl. Instrum. Methods Phys. Res. A 604, 133 (2009). 
${ }^{16}$ C. Algora, I. Rey-Stolle, I. Garcia, B. Galiana, M. Baudrit, and J. R. Gonzalez, J. Sol. Energy Eng. 129, 336 (2007).

${ }^{17}$ A. R. Vearey-Roberts and D. A. Evans, Appl. Phys. Lett. 86, 072105 (2005).

${ }^{18}$ D. N. Gnoth, D. Wolfframm, A. Patchett, S. Hohenecker, D. R. T. Zahn, A. Leslie, I. T. McGovern, and D. A. Evans, Appl. Surf. Sci. 123-124, 120 (1998).

${ }^{19}$ D. P. Langstaff, in Sensors and Their Applications VII, edited by A. T. Augousti (Institute of Physics Publishing, Dublin, 1995), pp. 108-111.

${ }^{20}$ A. R. Vearey-Roberts et al., Appl. Surf. Sci. 234, 131 (2004).

${ }^{21}$ J. C. Buchholz and G. A. Somorjai, J. Chem. Phys. 66, 573 (1977).
${ }^{22}$ D. Guay, G. Tourillon, L. Gastonguay, J. P. Dodelet, K. W. Nebesny, N. R. Armstrong, and R. Garrett, J. Phys. Chem. 95, 251 (1991).

${ }^{23}$ K. K. Okudaira, H. Setoyama, H. Yagi, K. Mase, S. Kera, A. Kahn, and N. Ueno, J. Electron Spectrosc. Relat. Phenom. 137-140, 137 (2004).

${ }^{24}$ M. L. M. Rocco, K. H. Frank, P. Yannoulis, and E. E. Koch, J. Chem. Phys. 93, 6859 (1990).

${ }^{25}$ B. N. Holland, N. Peltekis, T. Farrelly, R. G. Wilks, G. Gavrila, D. R. T. Zahn, C. McGuinness, and I. T. McGovern, Phys. Status Solidi B 246, 1546 (2009).

${ }^{26}$ J. Stohr, NEXAFS Spectroscopy (Springer, New York, 1992). 